\title{
Reachability Analysis for N-Squared State Charts over a Boolean Semiring Applied to a Hysteretic Discrete Event Structural Control Model
}

\author{
Patrick M. Sain, Ying Shang and Michael K. Sain
}

\begin{abstract}
This paper presents reachability results for a class of discrete event systems modelled by industry-standard $\mathrm{N}$-squared diagrams. These diagrams represent state transition charts using Boolean variables and functions, and form the basis for the design of hierarchical discrete event controllers used to operate, monitor and protect complex servomechanism systems. The presence of Boolean variables is addressed by means of dynamical systems modelled over semirings. Four distinct system descriptions capture the very subtle transformation from the physical problem to "standard" reachability constructions. The conditions are in a state space form and permit analytical assessment of plant and closed-loop system properties prior to coding in software, simulation and laboratory testing, thus offering immense savings in verification time and cost.
\end{abstract}

\section{INTRODUCTION}

This paper builds on the state space discrete event modelling in [1] based upon $\mathrm{N}^{2}$ diagrams by applying the ideas in Massey and Sain [2] to obtain qualitative reachability criteria for a family of discrete event systems. The state space model presented in [1] has close parallels to classical linear system theory, except that because it describes logical dynamics, the model is defined over a Boolean semiring instead of a field such as the real numbers.

$\mathrm{N}$-squared diagrams are an industry standard for modelling complex discrete event system behavior, and provide a basis for the mode and fault control designs in autonomous and semi-autonomous systems. Their matrix-like structure readily leads to state representations, supporting qualitative assessment of discrete event control system properties.

A major question for discrete event control system (DECS) design is whether operational states exist that cannot be entered under any conditions, or if there are states that once entered, cannot be left. Present state of the art relies upon expensive laboratory testing and is often incomplete. The results herein detail a method for analytically approaching such questions for DECS described by $\mathrm{N}^{2}$ diagrams, greatly reducing the need for laboratory testing.

The sequel introduces $\mathrm{N}^{2}$ diagrams using an example arising from the application of a hysteretic magnetorheological damper (MRD) to a 3DOF structure to mitigate responses to seismic excitation [1]. Next, the algebraic tools

P. Sain is with Raytheon Company, P. O. Box 902, El Segundo, CA, 90245, USA, Email: pmsainaraytheon.com

Y. Shang's work is supported by 2004-2005 Birck Fellowship, Department of Electrical Engineering, University of Notre Dame, Notre Dame, IN 46556 USA. Email: yshang@nd.edu

M. Sain's work is supported by the Frank M. Freimann Chair in Electrical Engineering, Department of Electrical Engineering, University of Notre Dame, Notre Dame, IN 46556 USA. Email: avemaria@nd. edu for casting DECS models into a state space form over a Boolean semiring are presented. Finally, the results in [2] are extended to obtain the desired reachability criteria.

\section{II. $\mathrm{N}^{2}$ DIAGRAM DEFINITION USING AN MRD}

The state space discrete event model presented herein was motivated while investigating means of controlling a three degree of freedom (3DOF) structure during seismic excitation. The nonlinear controller considered utilized a hysteretic magnetorheological damper (MRD) to control the structure's effective damping, a so-called semi-passive approach. The equations of motion for the structure are detailed by Dyke et al. [3], [4], but only the discrete event portion of the model is presented here.

The hysteretic nature of the MRD is represented using a second-order modified Bouc-Wen hysteresis model [5] described by a differential equation of the form

$$
\begin{array}{ll}
\dot{z}=\left(a-b_{1} z^{2}\right) \dot{x}, & \operatorname{sgn} z=\operatorname{sgn} \dot{x}, \\
\dot{z}=\left(a+b_{2} z^{2}\right) \dot{x}, & \operatorname{sgn} z \neq \operatorname{sgn} \dot{x},
\end{array}
$$

where $a, b_{1}, b_{2}, x$ and $z$ are real scalars, $x$ represents displacement of the MRD and the first story, $z$ is a nonphysical variable representing the hysteretic portion of the restoring force applied by the MRD, and $a, b_{1}$ and $b_{2}$ are loopshaping parameters for the hysteresis. The state chart for the MRD model is shown in Fig. 1.

For a given model having a set of $n$ distinct operating conditions, let the corresponding discrete event model have $n$ unique states. The corresponding $\mathrm{N}^{2}$ diagram is an $n \times$ $n$ grid with the $n$ discrete states of the model placed on the squares on the main diagonal of the grid. The first or entrance state is placed in the upper left corner and the exit or final state is placed in the lower right corner. Intermediate states can be placed in any order along the main diagonal. Fig. 2 illustrates placement of the states in Fig. 1 into an $\mathrm{N}^{2}$ diagram (entries $\mathrm{A} 1, \mathrm{~B} 2, \mathrm{C} 3$ and $\left.\mathrm{D} 4\right)$.

Transitions are represented on the off-diagonal elements in an $\mathrm{N}^{2}$ diagram. Let $s_{i}, i=1, \ldots, n$, denote the discrete states. Each transition is uniquely associated with a single source state and a single destination state. Given a unique pair of states $\left(s_{i}, s_{j}\right)$, where $i \neq j$, let $s_{i}$ denote the source state, and $s_{j}$ denote the destination state. The transition associated with this pair, denoted $t_{i j}$, is placed in grid location $(i, j)$ of the $\mathrm{N}^{2}$ diagram. Fig. 2 illustrates placement of the transitions shown in the state chart in Fig. 1 into an $\mathrm{N}^{2}$ diagram.

Transitions in Fig. 1 having the same source and destination state are shown only for completeness' sake and 


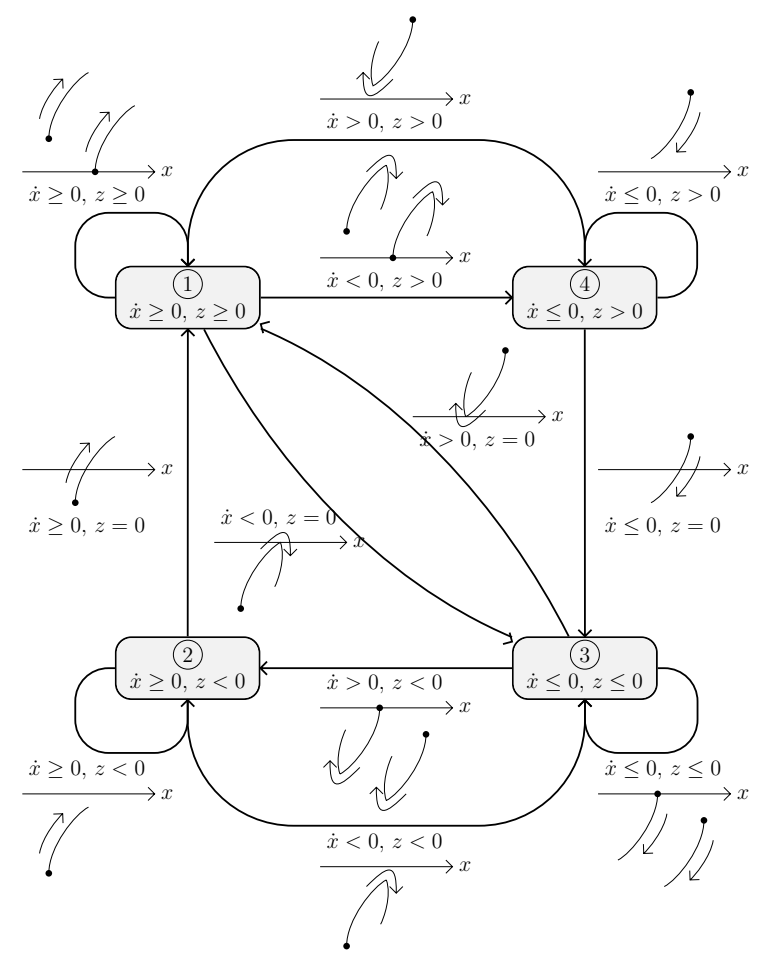

Fig. 1. State chart for a hysteretic MRD attached to a 3DOF structure.

\begin{tabular}{|c||c|c|c|c|}
\hline & 1 & 2 & 3 & 4 \\
\hline \hline $\mathrm{A}$ & $s_{1}$ & & $t_{13}$ & $t_{14}$ \\
\hline $\mathrm{B}$ & $t_{21}$ & $s_{2}$ & $t_{23}$ & \\
\hline $\mathrm{C}$ & $t_{31}$ & $t_{32}$ & $s_{3}$ & \\
\hline $\mathrm{D}$ & $t_{41}$ & & $t_{43}$ & $s_{4}$ \\
\hline
\end{tabular}

Fig. 2. $\mathrm{N}^{2}$ diagram for hysteretic MRD model.

are omitted in the $\mathrm{N}^{2}$ diagram. Also, this work assumes all relevant operating conditions of the modelled system are represented in the $\mathrm{N}^{2}$ diagram.

Let $s_{i}$ represent the state that corresponds to the present operating condition of the system. The system is then in state $s_{i}$, meaning the logical relations for state $s_{i}$ are true (see Table I). Equivalently, $s_{i}$ is active. The system changes to state $s_{j}$ if and only if an event occurs such that the conditions associated with transition $t_{i j}$ evaluate true (see Table II), assuming $t_{i j}$ exists; in this situation, transition $t_{i j}$ is said to be active. Note $\wedge$ and $\vee$ represent the logical and and or operations.

In the sequel, a serial process will be assumed. Extension of the model to parallel processes is reasonably straightforward, but beyond the scope of this paper. The following modelling assumptions for $\mathrm{N}^{2}$ diagrams are designed to facilitate control under fault conditions by eliminating ambiguity with respect to active states and transitions and also to admit a specialized state space representation [6].

Assumption 1 Each state is unique.

Assumption 2 At most one state is active at a time.

Assumption 3 At most one transition is active at a time.
TABLE I

MRD N ${ }^{2}$ STATE TABLE

\begin{tabular}{c|l}
\hline State & Description \\
\hline \hline$s_{1}$ & $(\dot{x} \geq 0) \wedge(z \geq 0)$ \\
\hline$s_{2}$ & $(\dot{x} \geq 0) \wedge(z<0)$ \\
\hline$s_{3}$ & $(\dot{x} \leq 0) \wedge(z \leq 0)$ \\
\hline$s_{4}$ & $(\dot{x} \leq 0) \wedge(z>0)$ \\
\hline
\end{tabular}

TABLE II

MRD N ${ }^{2}$ TRANSITION TABLE

\begin{tabular}{c|l}
\hline Transition & Description \\
\hline \hline$t_{13}$ & $(\dot{x}<0) \wedge(z=0)$ \\
\hline$t_{14}$ & $(\dot{x} \leq 0) \wedge(z>0)$ \\
\hline$t_{21}$ & $(\dot{x} \geq 0) \wedge(z=0)$ \\
\hline$t_{23}$ & $(\dot{x}<0) \wedge(z<0)$ \\
\hline$t_{31}$ & $(\dot{x}>0) \wedge(z=0)$ \\
\hline$t_{32}$ & $(\dot{x}>0) \wedge(z<0)$ \\
\hline$t_{41}$ & $(\dot{x}>0) \wedge(z>0)$ \\
\hline$t_{43}$ & $(\dot{x} \leq 0) \wedge(z=0)$ \\
\hline
\end{tabular}

Assumption 4 Ideally, the time interval over which a transition is active has measure zero.

If the system is in a given state $s_{i}$, and the logical relations associated with it evaluate false, then the system must change state. The new state is determined by examining the transitions $t_{i j}, 1 \leq j \leq n, i \neq j$. If, for example, transition $t_{i k}$ evaluates true, then the system has changed to state $s_{k}$. Changes requiring noneligible time intervals are modelled by creating a state that is active during the change.

At this point, a constructive approach for relating a specialized state space realization to the classical $\mathrm{N}^{2}$ diagram and associated state charts has been demonstrated. The appeal of the state space representation is that classical controls concepts like stability, reachability and detectability are readily formulated in a rigorous manner. The latter two concepts are of particular interest in discrete event control designs for operational mode and fault management. They provide a means of assessing whether a system can get into and out of operational modes as required, and also whether one can determine or observe what conditions triggered a particular response from the controller. In the sequel, results for reachability for such systems are presented, following the mathematical framework required to work within a Boolean semiring.

\section{Mathematical Preliminaries for Systems OVER A BOOLEAN SEMIRING}

A semigroup $(S, \square)$ is a set $S$ together with a binary operation $\square: S \times S \rightarrow S$ which is associative. A monoid $\left(M, \square, e_{M}\right)$ is a semigroup $(M, \square)$ with the unit element $e_{M}$ for $\square$, i.e. $e_{M} \square x=x \square e_{M}=x$ for all $x \in M$. A semiring $R=\left(R, \square, e_{R}, \circ, 1_{R}\right)$ is a set $R$ with two binary operations, box $\square$ and circle $\circ$, such that, $\left(R, \square, e_{R}\right)$ is a commutative monoid under $\square ;\left(R, \circ, 1_{R}\right)$ is a monoid under $\circ ; r \circ e_{R}=e_{R}=e_{R} \circ r$ for all $r \in R$; circle $\circ$ is distributive on both sides over box $\square$. A commutative semiring is a semiring in which the operator $\circ$ is commutative. A commutative semiring $R$ is idempotent if $a \square a=a$, for all $a \in R$. A Boolean set $B_{L}=(\{0,1\}, \vee, 0, \wedge, 1)$ with the logic operator OR $(\vee)$ and the logic operator AND $(\wedge)$ is a commutative idempotent semiring. Define a set $\bar{B}_{L}^{n}$ as the set of vectors with $n$ entries in $B_{L}$ and $G=\left\{\mathbf{e}_{1}, \cdots, \mathbf{e}_{n}\right\}$ as the subset of $\bar{B}_{L}^{n}$, where $\mathbf{e}_{1}=\left[\begin{array}{llll}1, & 0, \cdots, & 0\end{array}\right]^{T}$, 
$\mathbf{e}_{2}=[0,1, \cdots, 0]^{T}, \cdots$, and $\mathbf{e}_{n}=[0,0, \cdots, 1]^{T}$.

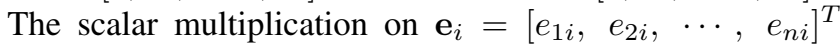
is defined as $k \mathbf{e}_{i}=\left[k \wedge e_{1 i}, k \wedge e_{2 i}, \cdots, k \wedge e_{n i}\right]^{T}$, where $k$ is in $B_{L}$ and $\mathbf{e}_{i}$ has only one nonzero entry $e_{i i}$, $\forall i \in\{1, \cdots, n\}$. The set $B_{L}^{n}$ is defined as the set of all linear combinations of elements $\mathbf{e}_{i}$ in $G$ under the logic OR operators, $B_{L}^{n}=\left\{\bigvee_{i=1}^{n} k_{i} \mathbf{e}_{i}: k_{i} \in B_{L}\right\}$. Notice that the elements in $B_{L}^{n}$ are sequences of arbitrary order of $\mathbf{e}_{i}$ in $G$, concatenated by the logic OR operators. We define a binary operator $\vee_{1}$ on $B_{L}^{n}$ as the ordinary OR operator, i.e. for any two elements $b$ and $b^{\prime}$ in $B_{L}^{n}$, where $b=\bigvee_{i=1}^{n} k_{i} \mathbf{e}_{i}$ and $b^{\prime}=\bigvee_{j=1}^{n} k_{i}^{\prime} \mathbf{e}_{j}$, we have

$$
b \vee_{1} b^{\prime}=\left(\bigvee_{i=1}^{n} k_{i} \mathbf{e}_{i}\right) \vee\left(\bigvee_{j=1}^{n} k_{j}^{\prime} \mathbf{e}_{j}\right)
$$

Therefore, $\left(B_{L}^{n}, \vee_{1}, \mathbf{0}\right)$ is a monoid and $\mathbf{0}$ is the zero vector. Notice that the vectors in any sequence of $B_{L}^{n}$ have no multiple nonzero entries, while the set $\bar{B}_{L}^{n}$ permits the multiple nonzero entries. The reason to distinguish these two sets is that the system model of the MRD only allows the state vector to have one nonzero entry. We can define a componentwise logic operator $\vee_{2}$ on $\bar{B}_{L}^{n}$. For any two elements $\bar{b}_{1}$ and $\bar{b}_{2}$ and $\bar{b}_{1}=\left[\bar{b}_{11}, \bar{b}_{21}, \cdots, \bar{b}_{n 1}\right]^{T}$ and $\bar{b}_{1}=\left[\bar{b}_{12}, \bar{b}_{22}, \cdots, \bar{b}_{n 2}\right]^{T}$, we have

$$
\bar{b}_{1} \vee_{2} \bar{b}_{2}=\left[\bar{b}_{11} \vee \bar{b}_{12}, \bar{b}_{21} \vee \bar{b}_{22}, \cdots, \bar{b}_{n 1} \vee \bar{b}_{n 2}\right]^{T} \text {. }
$$

Therefore, $\left(\bar{B}_{L}^{n}, \vee_{2}, \mathbf{0}\right)$ is also a monoid. Moreover, there exists an isomorphic map from $\bar{B}_{L}^{n}$ to $B_{L}^{n}$. This is because any vector $\bar{b}=\left[\bar{b}_{1}, \bar{b}_{2}, \cdots, \bar{b}_{n}\right]^{T}$ in $\bar{B}_{L}^{n}$ is isomorphic to an element in $B_{L}^{n}$ and vice versa, i.e.

$$
\bar{b} \cong\left[\begin{array}{c}
\bar{b}_{1} \\
0 \\
\vdots \\
0 \\
0
\end{array}\right] \vee\left[\begin{array}{c}
0 \\
\bar{b}_{2} \\
\vdots \\
0 \\
0
\end{array}\right] \vee \cdots \vee\left[\begin{array}{c}
0 \\
0 \\
\vdots \\
0 \\
\bar{b}_{n}
\end{array}\right] .
$$

If we have $A=\left(a_{i j}\right) \in \bar{B}_{L}^{n \times n}$ and $\mathbf{e}_{i} \in B_{L}^{n}$ with only one nonzero entry $e_{i i}$, then the matrix multiplication $\wedge_{1}$ : $B_{L}^{n} \rightarrow B_{L}^{n}$ can be defined as

$$
A \wedge_{1} \mathbf{e}_{i}=\bigvee_{l=1}^{n}\left[0, \cdots, a_{l i}, \cdots, 0\right]^{T} .
$$

Because an arbitrary element $b \in B_{L}^{n}$ is a linear combination of these generators $\mathbf{e}_{i}$, the matrix multiplication can be easily derived. If we have matrices $\bar{A}=\left(\bar{a}_{i j}\right) \in \bar{B}_{L}^{n \times n}$ and $\bar{b} \in \bar{B}_{L}^{n}$, then the componentwise matrix multiplication $\wedge_{2}: \bar{B}_{L}^{n} \rightarrow \bar{B}_{L}^{n}$ is defined by $\left(\bar{A} \wedge_{2} \bar{b}\right)_{i}=\bigvee_{k=1}^{n}\left(\bar{a}_{i k} \wedge \bar{b}_{k}\right)$ for $i=1, \cdots, m$. See Section V-C for a qualitative description of the difference between the ideas above.

\section{Physical System Representation}

\section{A. Physical System Model $\Sigma_{1}$}

Using the $\mathrm{N}$-squared diagram, the discrete event dynamics of the MRD can be written as a discrete-time linear system $\Sigma_{1}$ over a Boolean semiring $B_{L}$,

$$
x(k+1)=A(k) x(k) \vee_{1} b u(k),
$$

where $k$ represents the occurrence of the $k$-th event; the state $x$ is in $X=B_{L}^{n}$ and the control $u$ is in $U=B_{L} ; A \in \bar{B}_{L}^{n \times n}$ is a matrix mapping $A: X \rightarrow X$ and $b \in B_{L}^{n}$ is a matrix mapping $b: U \rightarrow X$. The matrix multiplication here is $\wedge_{1}$. The construction of $A(k)$ depends on the continuous dynamics, $\dot{x}_{p}=f\left(x_{p}, t, u_{p}\right)$, at each time $t_{k} \geq 0, t_{k} \in \mathbb{R}$, where $t_{k}$ is the occurrence time of the $k$-th event. Therefore, we need to evaluate the truth table of the $\mathrm{N}^{2}$ diagram to obtain $A(k)$. For instance, if, starting from a node $i$, the continuous state $x_{p}$ satisfies the logic conditions in node $s_{i}$ in the $\mathrm{N}^{2}$ diagram, then we enter 1 in $A_{i, i}(k)$ and enter 0 elsewhere. If the logic conditions at node $s_{i}$ are false, then we evaluate the transition condition $t_{i j}$ from node $i$ to $j$ in the $\mathrm{N}$-squared diagram. If it is true, then we enter 1 in $A_{j, i}(k)$ and enter 0 elsewhere. In other words, the matrix $A(k)$ not only depends on $k$ but also the continuous state vectors of the physical plant. The construction of $b$ can be determined by setting $b_{i}=1$ if $s_{i}$ is the initial node and all other entries in $b$ to zero. The input $u(k)=1$ for $k=0$, and is zero thereafter. The details of the construction of $A$ and $b$ matrices are in [6].

\section{B. Reachability of System $\Sigma_{1}$}

System $\Sigma_{1}$ models the discrete event behavior of the MRD and the matrices in system $\Sigma_{1}$ depend on continuous dynamics. Therefore, the reachability definition for $\Sigma_{1}$ is different from that in standard linear system theory [7]. We are given $x_{i}\left(k_{1}\right), x_{j}\left(k_{2}\right) \in X$, with nonzero entries only at the $i$-th and $j$-th rows, respectively; i.e. the node $i$ and the node $j$ are active in the $\mathrm{N}$-squared diagram, respectively. If $i \neq j$, then $x_{j}\left(k_{2}\right)$ is said to be 1-step reachable from $x_{i}\left(k_{1}\right)$ under the system $\Sigma_{1}$ if, for $t_{k_{1}} \leq t \leq t_{k_{1}+1}$, there exists a continuous control $u_{p}$ such that $x_{p}$ satisfies the transition condition $t_{i j}$ at time $t=t_{k_{1}+1}$, and no feasible transition condition prior to that time, so that $k_{2}=k_{1}+1$. If $j=i$, then, at the next event $k_{1}+1$, the continuous state $x_{p}$ has to satisfy the logic condition $s_{i}$ in the N-squared diagram, i.e. $s_{i}$ is true and $k_{2}=k_{1}+1$. This is equivalent to saying that the node $i$ is 1-step reachable from the node $i$ in the $\mathrm{N}^{2}$ diagram. We say that $x_{j}\left(k_{n+1}\right)$ is $n$-step reachable from $x_{i}\left(k_{1}\right)$ under the system $\Sigma_{1}$ if there exists a sequence of states

$$
\left\{x_{1}\left(k_{1}+1\right), x_{2}\left(k_{1}+2\right), \cdots, x_{n-1}\left(k_{1}+n-1\right)\right\} \subset X,
$$

in which $x_{1}\left(k_{1}+1\right)$ is 1 -step reachable from $x_{i}\left(k_{1}\right)$ and $x_{r+1}\left(k_{1}+r+1\right)$ is 1 -step reachable from $x_{r}\left(k_{1}+r\right)$, $r \in\{1,2, \cdots, n-2\}, x_{j}\left(k_{n+1}\right)$ is 1 -step reachable from $x_{n-1}\left(k_{1}+n-1\right)$, and $k_{n+1}=k_{1}+n$. The reachability of the system $\Sigma_{1}$ is equivalent to the reachability of nodes in the $\mathrm{N}^{2}$ diagram. A similar definition can also be found in the hybrid systems literature [8].

Because the entries of $A(k)$ depend on the continuous dynamics, we cannot compute the set of reachable states 
from an initial state without knowing the continuous states. However, if there exists a transition condition $t_{i j}$ in the $\mathrm{N}^{2}$ diagram, then there is a possibility the node $j$ can be reached from the node $i$. If there exists no transition condition at the intersection of the $i$-th row and the $j$-th column in the $\mathrm{N}^{2}$ diagram, then we can say the node $j$ can never be directly reached from the node $i$. Along this line, we are able to identify a subset $X^{*}$ of $X$, in which the states might be reached if the continuous dynamics can reach the transition regions. For any element $x$ in $X^{*}$, we can say that there exists a sequence of transition conditions from the initial state to $x$, and $x$ could possibly be reached, provided that the transition conditions are satisfied. For the state $x$ in $X$ but not in $X^{*}$, there is never a sequence of transitions which could drive an initial state to $x$. The subset of such states is denoted by $X / X^{*}$, which contains all unreachable states of $X$, in the sense of $\Sigma_{1}$.

\section{TIME-INVARIANT SySTEM REPRESENTATION}

\section{A. Simplified System Model $\Sigma_{2}$}

The system model for the physical MRD system is a time-varying system; so it is less convenient to study the reachability of such a system. If we assume that the continuous dynamics in the MRD are locally controllable, i.e. any transition conditions can be satisfied at appropriate time instants, then the system $\Sigma_{1}$ can be simplified into a time-invariant linear system $\Sigma_{2}$ over $B_{L}$,

$$
\hat{x}(k+1)=\hat{A} \hat{x}(k) \vee_{1} \hat{b} \hat{u}(k),
$$

where the state $\hat{x}$ is in $\hat{X}=B_{L}^{n}$ and the control $\hat{u} \in \hat{U}=$ $B_{L}$, the $\hat{A}$ matrix is the transpose of the truth table of the $\mathrm{N}$-squared diagram of this discrete-event model and $\hat{b}=b$.

\section{B. Reachability of System $\Sigma_{2}$}

Because the system $\Sigma_{2}$ is independent of the continuous dynamics, the reachability definition in ([9], p.158) can be extended to the time-invariant linear system $\Sigma_{2}$ over $B_{L}$. The sequence of reachable sets $\hat{X}_{i}$ under the control $\hat{u}$ of the system $\Sigma_{2}$ has the representation $\bigvee_{j=0}^{i-1} \hat{A}^{i-1-j} \hat{b}_{j}$, for the elements $\hat{b}_{j} \in \operatorname{Im} b$. We use the following construction

$$
\hat{A}\left(\bigvee_{j=0}^{i-1} \hat{A}^{i-1-j} \hat{b}_{j}\right) \vee_{1} \hat{b}_{j}=\bigvee_{j=0}^{i} \hat{A}^{i-j} \hat{b}_{j},
$$

to show that $\hat{A}_{*} \hat{X}_{i} \vee_{1} \operatorname{Im} \hat{b} \subset \hat{X}_{i+1}$, where $\hat{A}_{*} \hat{X}_{i}=\{x \mid x=$ $\left.\hat{A} x^{\prime}, x^{\prime} \in \hat{X}_{i}\right\}$. Conversely, if $\bigvee_{j=0}^{i} \hat{A}^{i-j} \hat{b}_{j} \in \hat{X}_{i+1}$, then

$$
\bigvee_{j=0}^{i} \hat{A}^{i-j} \hat{b}_{j}=\hat{A}\left(\bigvee_{j=0}^{i-1} \hat{A}^{i-1-j} \hat{b}_{j}\right) \vee_{1} \hat{b}_{i}
$$

Therefore, $\hat{X}_{i+1}=\hat{A}_{*} \hat{X}_{i} \vee_{1} \operatorname{Im} \hat{b}$. Then $\left(\left\{\hat{X}_{i} \mid i \in \mathbb{N}\right\}, \subset\right)$ is a chain and contained in the power set $\mathcal{P}(\hat{X})$ of $\hat{X}$, which is a complete lattice, $(\mathcal{P}(\hat{X}), \subset, \cap, \cup)$, so that the chain has a least upper bound $\hat{X}^{*}$, where $\hat{X}^{*}=\sup \left\{\hat{X}_{i} \mid i \in \mathbb{N}\right\}$.
The system $\Sigma_{2}$ is reachable if and only if $\hat{X}^{*}=\hat{X}$. If the system $\Sigma_{2}$ is not reachable, then some nodes in the $\mathrm{N}^{2}$ diagram cannot be reached. Note that the treatment in [9] assumes a sequence of inputs, and not just an impulse sequence. The set of elements in $\hat{X}$ but not in $\hat{X}^{*}$ is denoted by $\hat{X} / \hat{X}^{*}$, which is the set of unreachable states of the system $\Sigma_{2}$. Therefore, $X^{*} \subset \hat{X}^{*}$, which is an upper bound for $X^{*}$. However, if we consider impulse reachability, as reachability with an impulse sequence, with $\hat{X}_{\mathrm{imp}}^{*}$ the set of impulse-reachable states, then $X^{*}=\hat{X}_{\mathrm{imp}}^{*}$, as shown in the Appendix.

\section{Simplified System Model $\Sigma_{3}$}

Because the sets $B_{L}^{n}$ and $\bar{B}_{L}^{n}$ are isomorphic to each other, the system $\Sigma_{2}$ is also isomorphic to the following timeinvariant system $\Sigma_{3}$ over $B_{L}$,

$$
\bar{x}(k+1)=\bar{A} \bar{x}(k) \vee_{2} \bar{b} \bar{u}(k),
$$

where the $\bar{A}=\hat{A}$ matrix is the transpose of the truth table of the $\mathrm{N}^{2}$ diagram of the discrete-event model and $\bar{b}=\hat{b}=b$. The state $x$ is in $\bar{X}=\bar{B}_{L}^{n}$ and the control $\bar{u} \in \bar{U}=B_{L}$. The difference between the system $\Sigma_{3}$ and the system $\Sigma_{2}$ is that the state "strings" $\hat{x}$ in the system $\Sigma_{2}$ have no multiple nonzero entries, but the state $\bar{x}$ in the system $\Sigma_{3}$ can have multiple nonzero entries.

\section{Reachability of System $\Sigma_{3}$ and Nodes in the $N^{2}$ Diagram}

The reachability definition in [9] can also be extended to the system $\Sigma_{3}$. We are able to find an ascending chain of reachable sets $\bar{X}_{i}$. If the least upper bound $\bar{X}^{*}$ is equal to $\bar{X}$, then the system $\Sigma_{3}$ is reachable.

Proposition 1: If the following matrix of the system $\Sigma_{3}$,

$$
C_{r}(\bar{A}, \bar{b})=\left[\bar{b}, \bar{A} \wedge_{2} \bar{b}, \cdots, \bar{A}^{n-1} \wedge_{2} \bar{b}\right],
$$

has any zero rows, then the system $\Sigma_{3}$ is not reachable, i.e. $\bar{X}^{*} \neq \bar{X}$.

The matrix $C_{r}(\bar{A}, \bar{b})$ of the system $\Sigma_{3}$ evolves with a finite number of terms, because, if $\bar{X}_{i+1}=\bar{X}_{i}$, then $\bar{X}_{i+k}=\bar{X}_{i}$, for all $k=\{1,2, \cdots\}$, and there are only $n$ nodes in the N-squared diagram. Since not only $\hat{A}=\bar{A}$ and $\hat{b}=\bar{b}$, but also $\hat{X}$ is isomorphic to $\bar{X}$, we can verify that $\hat{X}^{*} \cong \bar{X}^{*}$. The reachability problems for these three systems are consistent with each other. The following proposition states this observation.

Proposition 2: If the matrix (6) of the system $\Sigma_{3}$ has zero rows, then both the system $\Sigma_{1}$ and the system $\Sigma_{2}$ are not reachable.

Proposition 3: If the matrix (6) of the system $\Sigma_{3}$ has zero rows, then the row numbers with zero entries are the unreachable node numbers in the $\mathrm{N}^{2}$ diagram; the row numbers with nonzero entries are the impulse reachable nodes in the $\mathrm{N}^{2}$ diagram if we assume that the transition conditions can be satisfied at corresponding time instants.

Remark: One must bear in mind that there are two notions here-reachability and impulse reachability. The former assumes a nonzero sequence of inputs, the latter 
an impulse input sequence. With respect to the matrix (6), in the former case we think of "linear combinations" of the columns; in the latter case, we consider the columns themselves.

\section{E. Simplified System Model $\Sigma_{4}$}

To simply the computation, we can use the following time-invariant system $\Sigma_{4}$ over the commutative semiring of non-negative real numbers, $\left(\mathbb{R}^{+},+, 0, \times, 1\right)$,

$$
\widetilde{x}(k+1)=\widetilde{A} \widetilde{x}(k)+\widetilde{b} \widetilde{u}(k),
$$

where $x \in \mathbb{R}^{+^{n}}$ and $u \in \mathbb{R}^{+}$, and the systems matrices $(\widetilde{A}, \widetilde{b})$ are the same as system matrices $(\bar{A}, \bar{b})$ in the system $\Sigma_{3}$. The following proposition gives us a feasible computational method in Matlab to verify which nodes in the $\mathrm{N}$ squared diagram are always not reachable.

Proposition 4: The matrix (6) of the system $\Sigma_{3}$ and the matrix of the system $\Sigma_{4}$,

$$
C_{r}(\widetilde{A}, \widetilde{b})=\left[\begin{array}{lllll}
\widetilde{b} & \widetilde{A} \times \widetilde{b} & \cdots & \widetilde{A}^{n-1} \times \widetilde{b}
\end{array}\right],
$$

have zero rows at the same time.

Proof: See Appendix.

\section{F. Example}

This simple example illustrates the results in this section. If we are considering a discrete-event system with the $\mathrm{N}$ squared diagram shown in Fig. 3 , then the systems $\Sigma_{1}$ and $\Sigma_{2}$ can be written as Eq. (3) and Eq. (4), where $x_{0}=[1,0,0,0,0]^{T}$. All $x(1)$, which might be reached, are $[1,0,0,0,0]^{T}$ or $[0,1,0,0,0]^{T}$. All $x(2)$, which might be reached, are $[1,0,0,0,0],[0,1,0,0,0]^{T}$ or $[0,0,0,1,0]^{T}$, so on and so forth. We find that all nodes in the $\mathrm{N}^{2}$ diagram, which might be reached, are nodes 1,2 and 4 .

\begin{tabular}{|c||c|c|c|c|c|}
\hline & 1 & 2 & 3 & 4 & 5 \\
\hline \hline$A$ & $s_{1}$ & $t_{12}$ & & & \\
\hline$B$ & & $s_{2}$ & & $t_{24}$ & \\
\hline$C$ & & & $s_{3}$ & & \\
\hline$D$ & $t_{41}$ & & & $s_{4}$ & \\
\hline$E$ & & & $t_{53}$ & & $s_{5}$ \\
\hline
\end{tabular}

Fig. 3. The N-squared diagram of a discrete event system

If we write the system $\Sigma_{3}$ using Eq. (5), where

$$
\bar{A}=\left(\begin{array}{ccccc}
1 & 0 & 0 & 1 & 0 \\
1 & 1 & 0 & 0 & 0 \\
0 & 0 & 1 & 0 & 1 \\
0 & 1 & 0 & 1 & 0 \\
0 & 0 & 0 & 0 & 1
\end{array}\right) \text { and } \bar{b}=\left(\begin{array}{l}
1 \\
0 \\
0 \\
0 \\
0
\end{array}\right),
$$

Then matrix $C_{r}(\bar{A}, \bar{b})$ of the system $\Sigma_{3}$ is

$$
C_{r}(\bar{A}, \bar{b})=\left(\begin{array}{ccccc}
1 & 1 & 1 & 1 & 1 \\
0 & 1 & 1 & 1 & 1 \\
0 & 0 & 0 & 0 & 0 \\
0 & 0 & 1 & 1 & 1 \\
0 & 0 & 0 & 0 & 0
\end{array}\right)
$$

which has two zero rows. Therefore, the nodes 3 and 5 are not reachable, which is consistent with the systems $\Sigma_{1}$ and $\Sigma_{2}$. Using Proposition 4 , the matrix $C_{r}(\widetilde{A}, \widetilde{b})$ of the system $\Sigma_{4}$ with the same $\bar{A}=\widetilde{A}$ and $\bar{b}=\widetilde{b}$ matrices is

$$
C_{r}(\widetilde{A}, \widetilde{b})=\left(\begin{array}{ccccc}
1 & 1 & 1 & 2 & 5 \\
0 & 1 & 2 & 3 & 5 \\
0 & 0 & 0 & 0 & 0 \\
0 & 0 & 1 & 3 & 6 \\
0 & 0 & 0 & 0 & 0
\end{array}\right),
$$

with the same zero rows, i.e. the nodes 3 and 5 are not reachable. This is also consistent with the conclusion from the system $\Sigma_{3}$.

\section{CONCLUSION}

A state realization of $\mathrm{N}^{2}$ diagrams over a Boolean semiring provides a means for mathematically analyzing qualitative properties of discrete event control systems (DECS). Of specific interest in this paper is the concept of reachability of linear systems modelled over the Boolean semiring. The generation of analytical qualitative results for such systems is a key feature because rigorous validation of the DECS' reachability is then possible. In particular, such concepts may be developed into a means of analyzing whether a DECS can get into and out of operational modes as required, without the necessity of conducting exhaustive (and potentially damaging in the case of faults and failures) simulations or tests.

\section{ACKNOWLEDGMENT}

The author gratefully acknowledges Raytheon Senior Technical Fellow Ron Cubalchini for his interest, support, constructive criticism and contributions to this work.

\section{REFERENCES}

[1] P. M. Sain, "Qualitative results for a hierarchical discrete event control paradigm applied to structures operating under nominal and fault Conditions," Proc. American Control Conference, 2004.

[2] J. L. Massey and M. K. Sain, "Codes, automata, and continuous systems: explicit interconnections," IEEE Trans. Auto. Control, AC12, pp. 644-650, 1967.

[3] S. J. Dyke, B. F. Spencer, Jr., M. K. Sain, and J. D. Carlson, "Seismic response reduction using magnetorheological dampers," Proc. IFAC World Congress, 1996.

[4] S. J. Dyke, B. F. Spencer, Jr., P. Quast and M. K. Sain, "Role of control-structure interaction in protective system design," ASCE J. Engrg. Mech., 121, pp. 322-338, 1995.

[5] P. M. Sain, M. K. Sain and B. F. Spencer, Jr., "Models for hysteresis and application to structural control," Proc. American Control Conference, pp. 16-20, 1997.

[6] P. M. Sain, "On application of precision servo mode and fault control strategies to actuator models for structural applications," Proc. American Control Conference, paper TP-07, 2002.

[7] A. N. Michel and P. J. Antsaklis, Linear Systems, McGraw-Hill, New York; 1997.

[8] T. A. Henzinger and Vlad Rusu, "Reachability verifiation for hybrid automata", Proc. First International Workshop on Hybrid Systems: Computation and Control (HSCC 98), Lecture Notes in Computer Science 1386, pp. 190-204, Springer-Verlag, 1998.

[9] M. K. Sain, Introduction to Algebraic System Theory, Academic Press, New York, 1981. 


\section{APPENDIX}

Proof of $X^{*}=\hat{X}_{\mathrm{imp}}^{*}$ : Define $N=\{1,2, \cdots, n\}$ as the set of discrete nodes, $O(i)$ as the set of nodes with nonempty entries in the $i$-th row of the N-squared diagram, i.e. $O(i)=\left\{j \in N \mid\right.$ if $j \neq i, \exists t_{i j}$; if $\left.j=i, \exists s_{i}\right\}$. If $O(i)=\left\{j_{1}, j_{2}, \cdots, j_{n_{i}}\right\}$, then the cardinality of $O(i)$ is $n_{i}$. Due to the construction of the $\mathrm{N}^{2}$ diagram, in which node 1 is always the initial active node, $b=\bar{b}=[1,0, \cdots, 0]^{T}$. All possible states in $x(1)$ of the system $\Sigma_{1}$ under the impulse input sequence are

$$
\begin{aligned}
x(1) & =\bigvee_{j \in O(1)} j\left(\begin{array}{cccc}
0 & 0 & \cdots & 0 \\
\vdots & \vdots & \cdots & \vdots \\
1 & 0 & \cdots & 0 \\
\vdots & \vdots & \cdots & \vdots \\
0 & 0 & \cdots & 0
\end{array}\right)\left(\begin{array}{c}
1 \\
\vdots \\
0 \\
\vdots \\
0
\end{array}\right) \vee_{1}\left(\begin{array}{c}
1 \\
\vdots \\
0 \\
\vdots \\
0
\end{array}\right) \\
& =\bigvee_{j \in O(1)}\left(0, \cdots, \alpha_{j 1}, \cdots, 0\right)^{T},
\end{aligned}
$$

where $\alpha_{j 1}=1$ if and only if $j \in O(1)$. Notice that node 1 is always a possible active node because there exists a possible self loop in the $\mathrm{N}^{2}$ diagram. The $\hat{x}(1)$ is

$$
\begin{aligned}
\hat{x}(1) & =\left(\begin{array}{ccc}
\hat{a}_{11} & \cdots & \hat{a}_{1 n} \\
\vdots & \cdots & \vdots \\
\hat{a}_{n 1} & \cdots & \hat{a}_{n n}
\end{array}\right)\left(\begin{array}{c}
1 \\
\vdots \\
0
\end{array}\right) \vee_{1}\left(\begin{array}{c}
1 \\
\vdots \\
0
\end{array}\right) \\
& =\bigvee_{j=1}^{n}\left(0, \cdots, \hat{a}_{j 1}, \cdots, 0\right)^{T},
\end{aligned}
$$

where $\hat{a}_{j 1}=1$ if and only if $j \in O(1)$. Therefore, $x(1)=\hat{x}(1)$. By induction, if $x(k)=\hat{x}(k), x(k)=$ $\hat{x}(k)=\bigvee_{1}\left(0, \cdots, x_{i k}, \cdots, 0\right)^{T}$, and $i \in I_{k}=$ $\left\{i_{1}, i_{2}, \cdots, i_{n_{k}}\right\} \subset N$ implies $x_{i k}=1$; otherwise $x_{i k}=0$. The next state $x(k+1)$ can be computed from $x(k+1)=$ $A(k) x(k)=\bigvee_{i \in I_{k}} A(k)\left(0 \cdots x_{i k} \cdots 0\right)^{T}$ and is equal to

$$
\begin{aligned}
& =\bigvee_{i \in I_{k}} \bigvee_{j \in O(i)} j\left(\begin{array}{ccccc}
0 & \cdots & 0 & \cdots & 0 \\
\vdots & \cdots & \vdots & \cdots & \vdots \\
0 & \cdots & 1 & \cdots & 0 \\
\vdots & \cdots & \vdots & \cdots & \vdots \\
0 & \cdots & 0 & \cdots & 0
\end{array}\right)\left(\begin{array}{c}
0 \\
\vdots \\
x_{i k} \\
\vdots \\
0
\end{array}\right) \\
& =\bigvee_{i \in I_{k} j \in O(i)} \bigvee_{1}\left(0, \cdots, \alpha_{j i}, \cdots, 0\right)^{T}
\end{aligned}
$$

where $\alpha_{j i}=1$ if and only if $j \in O(i)$. The next state $\hat{x}(k+1)=\hat{A} \hat{x}(k)=\bigvee_{i \in I_{k}} \hat{A}\left(0 \cdots x_{i k} \cdots 0\right)^{T}$ is equal to

$$
\bigvee_{i \in I_{k}}\left(\begin{array}{ccccc}
\hat{a}_{11} & \cdots & \hat{a}_{1 i} & \cdots & \hat{a}_{1 n} \\
\vdots & \cdots & \vdots & \cdots & \vdots \\
\hat{a}_{j 1} & \cdots & \hat{a}_{j i} & \cdots & \hat{a}_{j n} \\
\vdots & \cdots & \vdots & \cdots & \vdots \\
\hat{a}_{n 1} & \cdots & \hat{a}_{n i} & \cdots & \hat{a}_{n n}
\end{array}\right)\left(\begin{array}{c}
0 \\
\vdots \\
x_{i k} \\
\vdots \\
0
\end{array}\right)
$$

$$
=\bigvee_{i \in I_{k}} \bigvee_{1 \in O(i)}\left(0, \cdots, \hat{a}_{j i}, \cdots, 0\right)^{T}
$$

where $\hat{a}_{j i}=1$ if and only if $j \in O(i)$. Therefore, we have that $X^{*}=\hat{X}_{\text {imp }}^{*} \diamond$

Proof of Proposition 4: We will prove that each column of the matrices (6) and (8) has zero rows at the same time. Starting from the 1 -st column, because $\widetilde{b}=\bar{b}=$ $[1,0, \cdots, 0]^{T}$, they have the same zero rows. Since $\bar{A}=\widetilde{A}$, we examine the second column $\bar{A} \wedge_{2} \bar{b}$ and $\widetilde{A} \times \widetilde{b}$ and obtain that $\bar{A} \wedge_{2} \bar{b}=\widetilde{A} \times \widetilde{b}$ which is equal to

$$
\left(\begin{array}{ccc}
\bar{a}_{11} & \cdots & \bar{a}_{1 n} \\
\vdots & \cdots & \vdots \\
\bar{a}_{n 1} & \cdots & \bar{a}_{n n}
\end{array}\right)\left(\begin{array}{c}
1 \\
\vdots \\
0
\end{array}\right)=\left(\begin{array}{c}
\bar{a}_{11} \\
\vdots \\
\bar{a}_{n 1}
\end{array}\right) .
$$

Therefore, they have zero rows at the same time. By induction, if $\bar{A}^{k} \wedge_{2} \bar{b}$ and $\widetilde{A}^{k} \times \widetilde{b}$ have zero rows at the same time, then we need to show that $\bar{A}^{k+1} \wedge_{2} \bar{b}$ and $\widetilde{A}^{k+1} \times \widetilde{b}$ also have zero rows at the same time. Assume $\bar{A}^{k} \wedge_{2} \bar{b}=$ $\left(\bar{b}_{1 k}, \cdots, \bar{b}_{n k}\right)$ and $\widetilde{A}^{k} \times \widetilde{b}=\left(\widetilde{b}_{1 k}, \cdots, \widetilde{b}_{n k}\right)$. The $i$ th rows, where $i \in I_{k}=\left\{i_{1}, \cdots, i_{n_{k}}\right\} \subset N$, of the vector $\bar{A}^{k} \wedge_{2} \bar{b}$ and $\widetilde{A}^{k} \times \widetilde{b}$ are both non-zero; otherwise the rows are both zero. Then $\bar{A}^{k+1} \wedge_{2} \bar{b}=\bar{A} \bigvee_{i \in I_{k}}\left(\begin{array}{lllll}0 & \cdots & \bar{b}_{i k} & \cdots & 0\end{array}\right)^{T}=$ $\bigvee_{i \in I_{k}} \bar{A}\left(0 \cdots \bar{b}_{i k} \cdots\right)^{T}$ which is equal to

$$
\begin{aligned}
& \bigvee_{2}\left(\begin{array}{ccccc}
\bar{a}_{11} & \cdots & \bar{a}_{1 i} & \cdots & \bar{a}_{1 n} \\
\vdots & \cdots & \vdots & \cdots & \vdots \\
\bar{a}_{j 1} & \cdots & \bar{a}_{j i} & \cdots & \bar{a}_{j n} \\
\vdots & \cdots & \vdots & \cdots & \vdots \\
\bar{a}_{n 1} & \cdots & \bar{a}_{n i} & \cdots & \bar{a}_{n n}
\end{array}\right)\left(\begin{array}{c}
0 \\
\vdots \\
\bar{b}_{i k} \\
\vdots \\
0
\end{array}\right) \\
= & \bigvee_{i \in I_{k}}\left(\bar{a}_{1 i} \vee \bar{b}_{i k}, \cdots, \bar{a}_{j i} \vee \bar{b}_{i k}, \cdots, \bar{a}_{n i} \vee \bar{b}_{i k}\right)^{T} \\
= & \bigvee_{i \in I_{k}}\left(\bar{a}_{1 i}, \cdots, \bar{a}_{j i} \cdots, \bar{a}_{n i}\right)^{T},
\end{aligned}
$$

where $\bar{a}_{j i}$ is non-zero if and only if $j \in O(i)$; $\widetilde{A}^{k+1} \times \widetilde{b}=\widetilde{A} \sum_{i \in I_{k}}\left(\begin{array}{llllll}0 & \cdots & \widetilde{b}_{i k} & \cdots & 0)^{T}=\end{array}\right.$ $\sum_{i \in I_{k}} \widetilde{A}\left(0 \cdots \widetilde{b}_{i k} \cdots 0\right)^{T}$ which is equal to

$$
\begin{aligned}
& \sum_{i \in I_{k}}\left(\begin{array}{ccccc}
\widetilde{a}_{11} & \cdots & \widetilde{a}_{1 i} & \cdots & \widetilde{a}_{1 n} \\
\vdots & \cdots & \vdots & \cdots & \vdots \\
\widetilde{a}_{j 1} & \cdots & \widetilde{a}_{j i} & \cdots & \widetilde{a}_{j n} \\
\vdots & \cdots & \vdots & \cdots & \vdots \\
\widetilde{a}_{n 1} & \cdots & \widetilde{a}_{n i} & \cdots & \widetilde{a}_{n n}
\end{array}\right)\left(\begin{array}{c}
0 \\
\vdots \\
\widetilde{b}_{i k} \\
\vdots \\
0
\end{array}\right) \\
= & \sum_{i \in I_{k}}\left(\widetilde{a}_{1 i} \times \widetilde{b}_{i k}, \cdots, \widetilde{a}_{j i} \times \widetilde{b}_{i k}, \cdots, \widetilde{a}_{n i} \times \widetilde{b}_{i k}\right)^{T},
\end{aligned}
$$

where $\widetilde{a}_{j i} \times \widetilde{b}_{i k} \neq 0$ if and only if $j \in O(i)$. Thus, $\bar{A}^{k+1} \wedge_{2} \bar{b}$ and $\widetilde{A}^{k+1} \times \widetilde{b}$ have zero or non-zero rows at the same time. Thus, the two matrices $C_{r}(\bar{A}, \bar{b})$ and $C_{r}(\widetilde{A}, \widetilde{b})$ have the zero or non-zero rows at the same time. $\diamond$ 